\title{
INCLUSIONS OF TERNARY RINGS OF OPERATORS AND CONDITIONAL EXPECTATIONS
}

\author{
PEKKA SALMI AND ADAM SKALSKI
}

\begin{abstract}
It is shown that if $T$ is a ternary ring of operators (TRO), $X$ is a nondegenerate sub-TRO of $T$ and there exists a contractive idempotent surjective map $P: T \rightarrow X$ then $P$ has a unique, explicitly described extension to a conditional expectation between the associated linking algebras. A version of the result for $W^{*}$-TROs is also presented and some applications mentioned.
\end{abstract}

Ternary rings of operators (TROs) are norm-closed subspaces of operators acting between Hilbert spaces which are in addition stable under the ternary product: $(a, b, c) \mapsto a b^{*} c$. They form a special class of concrete operator spaces and in fact possess also an abstract characterisation in terms of the operator-space-theoretic properties ([NeRu02]). A fundamental tool for their study is the construction of a so-called linking algebra, which is a particular $C^{*}$-algebra containing the TRO in question as a corner. As TROs share many properties with their associated linking algebras, the construction facilitates the application of operator algebraic techniques to the analysis of objects which are not algebras themselves (examples of this type can be found in [EOR01] and [KaRu02]).

In this note we follow this philosophy and apply it to the analysis of a sub-TRO $X$ of a given TRO $T$, showing that if $X$ is expected, i.e. there exists a contractive idempotent map from $T$ onto $X$ and a natural nondegeneracy condition is satisfied, then the map in question extends to a (unique, explicitly described) conditional expectation between the respective linking algebras. The proof is based on certain matrix calculations and the formula for the norm of an element in a left (or right) linking algebra due to Hamana (Ham99). An analogous result is valid for $W^{*}$ TROs, i.e. TROs which are closed in the weak*-topology, with the respective maps being normal. We indicate also some applications.

\section{Notation AND PRELIMINARIES}

For Hilbert spaces $H$ and $K$, let $\mathrm{B}(H, K)$ denote the set of all bounded operators from $H$ to $K$. A TRO (i.e. ternary ring of operators) is a closed subspace $T \subseteq$ $\mathrm{B}(H, K)$ such that $T T^{*} T \subseteq T$. In this case, the norm closed linear spans $C:=$ $\left\langle T T^{*}\right\rangle$ and $D:=\left\langle T^{*} T\right\rangle$ are $C^{*}$-subalgebras of $\mathrm{B}(K)$ and $\mathrm{B}(H)$, respectively, and these $C^{*}$-algebras act non-degenerately on $T$ (i.e. $T=C T=T D$; note also that for subsets of normed spaces we will always use angled brackets to denote closed linear spans). The norms on these so-called left and right linking algebras $C$ and $D$ are determined by the module actions: for every $c \in C$,

$$
\|c\|=\sup \{\|c t\| ; t \in T,\|t\| \leq 1\}
$$

2010 Mathematics Subject Classification. Primary 46L07, Secondary 17C65, 47L05.

Key words and phrases. Ternary ring of operators, linking algebra, expectation. 
and the analogous equation holds for every $d \in D$. (See Hamana Ham99, Lemma 2.3.) The linking algebra of $T$ is the $C^{*}$-algebra

$$
\mathrm{A}_{T}:=\left(\begin{array}{cc}
\left\langle T T^{*}\right\rangle & T \\
T^{*} & \left\langle T^{*} T\right\rangle
\end{array}\right) \subseteq \mathrm{B}(K \oplus H)
$$

Suppose that $T$ is a TRO and $P: T \rightarrow X$ is a completely contractive projection onto a sub-TRO $X \subseteq T$. As follows from the work of Youngson (You83, Corollary 3 ), the map $T$ is then a TRO conditional expectation in the sense that

$$
\begin{aligned}
& P\left(a x^{*} y\right)=P(a) x^{*} y \\
& P\left(x a^{*} y\right)=x P(a)^{*} y \quad \text { for every } a \in T, x, y \in X . \\
& P\left(x y^{*} a\right)=x y^{*} P(a)
\end{aligned}
$$

Effros, Ozawa and Ruan showed in [EOR01, Theorem 2.5, that already a contractive projection onto a sub-TRO is necessarily a completely contractive TRO conditional expectation.

We say that the sub-TRO $X \subseteq T$ is nondegenerate if

$$
\left\langle X T^{*} T\right\rangle=T \quad \text { and } \quad\left\langle T T^{*} X\right\rangle=T .
$$

Since $\left\langle T T^{*} T\right\rangle=T$, it follows from these identities that in fact

$$
\left\langle X X^{*} T\right\rangle=T \quad\left\langle T X^{*} X\right\rangle=T \quad\left\langle X T^{*}\right\rangle=\left\langle T T^{*}\right\rangle \quad\left\langle T^{*} X\right\rangle=\left\langle T^{*} T\right\rangle .
$$

The following proposition is a straighforward consequence of the above identities.

Proposition 1.1. A sub-TRO $X \subseteq T$ is nondegenerate if and only if the $C^{*}$ subalgebra $\mathrm{A}_{X}$ is nondegenerate in $\mathrm{A}_{T}$.

For a map $P: T \rightarrow X$ and $t \in T$ we let $P^{\dagger}: T^{*} \rightarrow X^{*}$ be defined by the formula $P^{\dagger}(t)=P\left(t^{*}\right)^{*}$. If $X \subset T$ is nondegenerate and a TRO conditional expectation from $T$ onto $X$ exists, we will say that $X$ is an expected sub-TRO of $T$.

\section{MAIN RESUlt}

This section contains the main result of the paper. Observe that the construction in the proof follows (and in a sense generalises) that given in Proposition 2.1 (iv) of [Ham99.

Theorem 2.1. Suppose that $T$ is a TRO and $P: T \rightarrow X$ is a contractive idempotent map onto a nondegenerate sub-TRO $X \subseteq T$. Then there is a $C^{*}$-algebra conditional expectation from the linking algebra $\mathrm{A}_{T}$ onto the linking algebra $\mathrm{A}_{X}$

$$
E:=\left(\begin{array}{cc}
P P^{\dagger} & P \\
P^{\dagger} & P^{\dagger} P
\end{array}\right):\left(\begin{array}{cc}
\left\langle T T^{*}\right\rangle & T \\
T^{*} & \left\langle T^{*} T\right\rangle
\end{array}\right) \rightarrow\left(\begin{array}{cc}
\left\langle X X^{*}\right\rangle & X \\
X^{*} & \left\langle X^{*} X\right\rangle
\end{array}\right)
$$

where the corner maps satisfy

$$
P P^{\dagger}\left(\sum_{i=1=}^{n} a_{i} x_{i}^{*}\right)=\sum_{i=1}^{n} P\left(a_{i}\right) x_{i}^{*} \quad \text { and } \quad P^{\dagger} P\left(\sum_{i=1}^{n} x_{i}^{*} a_{i}\right)=\sum_{i=1}^{n} x_{i}^{*} P\left(a_{i}\right)
$$

for $n \in \mathbb{N}, a_{1}, \ldots, a_{n} \in T$ and $x_{1}, \ldots, x_{n} \in X$. The extension is unique in the sense that if

$$
E^{\prime}=\left(\begin{array}{ll}
E_{11}^{\prime} & E_{12}^{\prime} \\
E_{21}^{\prime} & E_{22}^{\prime}
\end{array}\right)
$$

is another conditional expectation from $A_{T}$ onto $A_{X}$ such that $E_{12}^{\prime}=P$, then $E^{\prime}=E$. 
Proof. Note first that Theorem 2.5 of [EOR01] implies that $P: T \rightarrow X$ is a TRO conditional expectation.

By nondegeneracy, finite sums $a=\sum_{i} b_{i} x_{i}^{*}$ where $b_{i} \in T$ and $x_{i} \in X$ are dense in $\left\langle T T^{*}\right\rangle$. For such $a$, define

$$
P P^{\dagger}(a)=\sum_{i} P\left(b_{i}\right) x_{i}^{*}
$$

(that $P P^{\dagger}$ is in fact well defined follows shortly). By (1)

$$
\begin{aligned}
\left\|\sum_{i} P\left(b_{i}\right) x_{i}^{*}\right\| & =\sup \left\{\left\|\sum_{i} P\left(b_{i}\right) x_{i}^{*} y\right\| ; y \in X,\|y\| \leq 1\right\} \\
& =\sup \left\{\left\|P\left(\sum_{i} b_{i} x_{i}^{*} y\right)\right\| ; y \in X,\|y\| \leq 1\right\} \leq\left\|\sum_{i} b_{i} x_{i}^{*}\right\|
\end{aligned}
$$

because $P$ is a contractive TRO conditional expectation. It follows that $P P^{\dagger}$ is well defined and bounded with norm 1 . Hence we may extend $P P^{\dagger}$ to a map from $\left\langle T T^{*}\right\rangle$ into $\left\langle X X^{*}\right\rangle$, and it is onto because on $\left\langle X X^{*}\right\rangle$ the map $P P^{\dagger}$ is clearly the identity. Consequently, $P P^{\dagger}$ is a $C^{*}$-algebra conditional expectation from $\left\langle T T^{*}\right\rangle$ onto $\left\langle X X^{*}\right\rangle$.

Similarly, one can check that the map $P^{\dagger} P$ is a $C^{*}$-algebra conditional expectation from $\left\langle T^{*} T\right\rangle$ onto $\left\langle X^{*} X\right\rangle$.

The map

$$
E=\left(\begin{array}{cc}
P P^{\dagger} & P \\
P^{\dagger} & P^{\dagger} P
\end{array}\right):\left(\begin{array}{cc}
\left\langle T T^{*}\right\rangle & T \\
T^{*} & \left\langle T^{*} T\right\rangle
\end{array}\right) \rightarrow\left(\begin{array}{cc}
\left\langle X X^{*}\right\rangle & X \\
X^{*} & \left\langle X^{*} X\right\rangle
\end{array}\right)
$$

is a well-defined projection from $\mathrm{A}_{T}$ onto its $C^{*}$-subalgebra $\mathrm{A}_{X}$. To prove that this map is a conditional expectation, it is enough to show that it is contractive. We shall do this using the module norm identity (11) again, this time applied to the TRO

$$
\left(\begin{array}{c}
X \\
\left\langle X^{*} X\right\rangle
\end{array}\right) \subseteq B(H, K \oplus H)
$$

and its left linking algebra $\mathrm{A}_{X}$. Let $t_{i}, r, s, v_{j} \in T, x_{i}, y_{j}, z \in X$ and $a \in\left\langle X^{*} X\right\rangle$. Then

$$
\begin{aligned}
\left(\begin{array}{cc}
P P^{\dagger}\left(\sum_{i} t_{i} x_{i}^{*}\right) & P(r) \\
P^{\dagger}\left(s^{*}\right) & P^{\dagger} P\left(\sum_{j} y_{j}^{*} v_{j}\right)
\end{array}\right)\left(\begin{array}{c}
z \\
a
\end{array}\right) & =\left(\begin{array}{c}
\sum_{i} P\left(t_{i}\right) x_{i}^{*} z+P(r) a \\
P(s)^{*} z+P^{\dagger} P\left(\sum_{j} y_{j}^{*} v_{j}\right) a
\end{array}\right) \\
& =\left(\begin{array}{c}
P\left(\sum_{i} t_{i} x_{i}^{*} z+r a\right) \\
P^{\dagger} P\left(s^{*} z+\sum_{j} y_{j}^{*} v_{j} a\right)
\end{array}\right)
\end{aligned}
$$

where the final step needs some explanation: note that $P$ is a TRO conditional expectation, $P^{\dagger} P$ is a $C^{*}$-algebra conditional expectation, and that

$$
P(t)^{*} P(u)=P^{\dagger} P\left(P(t)^{*} u\right)=P^{\dagger} P\left(t^{*} P(u)\right)
$$

for every $t, u \in T$ essentially by definition.

In light of (3) and (11), it is enough to show that the map

$$
\left(\begin{array}{c}
P \\
P^{\dagger} P
\end{array}\right)
$$


is contractive. Using (4) again, we have for every $t \in T$ and $b \in\left\langle T^{*} T\right\rangle$ that

$$
\begin{aligned}
\left\|\left(\begin{array}{c}
P(t) \\
P^{\dagger} P(b)
\end{array}\right)\right\|^{2} & =\left\|P(t)^{*} P(t)+P^{\dagger} P(b)^{*} P^{\dagger} P(b)\right\|=\left\|P^{\dagger} P\left(P(t)^{*} t+P^{\dagger} P(b)^{*} b\right)\right\| \\
& \leq\left\|\left(\begin{array}{c}
P(t) \\
P^{\dagger} P(b)
\end{array}\right)^{*}\left(\begin{array}{l}
t \\
b
\end{array}\right)\right\| \leq\left\|\left(\begin{array}{c}
P(t) \\
P^{\dagger} P(b)
\end{array}\right)\right\|\left\|\left(\begin{array}{l}
t \\
b
\end{array}\right)\right\| .
\end{aligned}
$$

Hence

as required.

$$
\left\|\left(\begin{array}{c}
P(t) \\
P^{\dagger} P(b)
\end{array}\right)\right\| \leq\left\|\left(\begin{array}{l}
t \\
b
\end{array}\right)\right\|
$$

Finally, we prove the uniqueness statement. For $t \in T$ and $x \in X$,

$$
\left(\begin{array}{cc}
E_{11}^{\prime}\left(t x^{*}\right) & 0 \\
0 & 0
\end{array}\right)=E^{\prime}\left(\left(\begin{array}{ll}
0 & t \\
0 & 0
\end{array}\right)\left(\begin{array}{cc}
0 & 0 \\
x^{*} & 0
\end{array}\right)\right)=\left(\begin{array}{cc}
E_{12}^{\prime}(t) x^{*} & 0 \\
0 & 0
\end{array}\right)
$$

Hence $E_{11}^{\prime}\left(t x^{*}\right)=P(t) x^{*}=P P^{\dagger}\left(t x^{*}\right)$. By nondegeneracy $E_{11}^{\prime}=P P^{\dagger}$. Equalities for the other entry maps follow similarly.

Note that the main part of the above proof provides an alternative direct proof of a special case of Theorem 2.5 of [EOR01, namely it shows that a contractive TRO conditional expectation onto a non-degenerate sub-TRO is necessarily completely bounded (Theorem 2.5 of EOR01] was used in the above proof only to deduce the algebraic properties of the TRO-conditional expectation $P$ from its contractivity, so the proof shows that contractivity together with the algebraic properties imply complete contractivity). We also have the following immediate corollary.

Corollary 2.2. Let $T$ be a TRO and $X \subseteq T$ a nondegenerate sub-TRO. If $E$ : $\mathrm{A}_{T} \rightarrow \mathrm{A}_{X}$ is a conditional expectation (onto $\mathrm{A}_{X}$ ) and $E$ maps $T \subset A_{T}$ into $T$, then $E$ is of the form (2) for $P:=\left.E\right|_{T}$.

A direct motivation to study the construction described in Theorem 2.1 came from the desire to characterise contractive idempotent functionals on $C_{0}(\mathbb{G})$, the $C^{*}$ algebra of continuous, vanishing at infinity functions on a locally compact quantum group $\mathbb{G}$, in terms of certain subspaces of $C_{0}(\mathbb{G})$ (which turn out to be TROs). We refer the reader to NSSS12 for the details, noting that the explicit form of the conditional expectation established in Theorem 2.1 plays a significant role there.

On the other hand Theorem 2.1 together with the techniques developed in KaRu02, showing that a TRO shares for example many approximation properties with its linking algebra, allows us for example to show immediately that Lancenuclearity (in the terminology of [KaRu02]) passes to expected sub-TROs.

Corollary 2.3. Let $T$ be a TRO and $X \subset T$ an expected nondegenerate sub-TRO. If $T$ is Lance-nuclear then so is $X$.

Proof. This follows from Theorem 2.1, Theorem 6.1 of [KaRu02] and the fact that nuclearity of $C^{*}$-algebras is preserved under taking conditional expectations.

\section{EXTENSION TO $W^{*}$-TROS}

In this section we present a version of Theorem 2.1 for $W^{*}$-TROs. A $W^{*}$ TRO is a TRO $T \subseteq \mathrm{B}(H, K)$ that is closed under the weak* topology. We may assume without loss of generality that $T$ is nondegenerately represented, i.e. that $\langle T H\rangle=K$ and $\left\langle T^{*} K\right\rangle=H$. A $W^{*}$-sub-TRO $X \subseteq T$ is nondegenerate if the linear 
spans of $X T^{*} T$ and $T T^{*} X$ are weak*-dense in $T$. The next proposition connects this property to $X$ itself being nondegenerately represented.

Proposition 3.1. Suppose that $T$ is a nondegenerately represented $W^{*}-T R O$ in $\mathrm{B}(H, K) . A W^{*}$-sub-TRO $X \subseteq T$ is nondegenerate if and only if $X$ is nondegenerately represented in $\mathrm{B}(H, K)$.

Proof. If $X$ is nondegenerate, then the linear spans of $X T^{*} T$ and $T T^{*} X$ are (as convex sets) so-dense in $T$, so a simple calculation allows us to approximate elements in $T H$ (respectively, in $T^{*} K$ ) by elements in $X H$ (respectively, in $X^{*} K$ ).

Conversely, if $X$ is nondegenerately represented in $\mathrm{B}(H, K)$, then $\left(X X^{*}\right)^{\prime \prime}$ is a (nondegenerate) von Neumann algebra on $K$ and hence contains the identity operator on $K$. It follows that $T$ is contained in the weak*-closed linear span of $X X^{*} T$. Similarly, $T$ is contained in the weak*-closed linear span of $T X^{*} X$, and so $X$ is nondegenerate in $T$.

We need one more lemma which may be viewed as a version of the Kaplansky theorem for nondegenerate $W^{*}$-sub-TROs.

Lemma 3.2. Suppose that $T$ is a nondegenerately represented $W^{*}-T R O$ in $\mathrm{B}(H, K)$ and that $X \subseteq T$ is a nondegenerate $W^{*}$-sub-TRO. For every $u$ in $\left(T T^{*}\right)^{\prime \prime}$ there is a net $\left(a_{\alpha}\right)$ in $\operatorname{span}\left(T X^{*}\right)$ such that $\left(a_{\alpha}\right)$ is bounded by $\|u\|$ and $a_{\alpha} \rightarrow u$ weak* ${ }^{*}$. Similar statements hold when $\left(T T^{*}\right)^{\prime \prime}$ is replaced by

$$
\left(T^{*} T\right)^{\prime \prime} \quad\left(\text { respectively, }\left(\begin{array}{cc}
\left(T T^{*}\right)^{\prime \prime} & T \\
T^{*} & \left(T^{*} T\right)^{\prime \prime}
\end{array}\right)\right)
$$

and $\operatorname{span}\left(T X^{*}\right)$ is replaced by

$$
\operatorname{span}\left(X^{*} T\right) \quad\left(\text { respectively, }\left(\begin{array}{cc}
\operatorname{span}\left(T X^{*}\right) & T \\
T^{*} & \operatorname{span}\left(X^{*} T\right)
\end{array}\right)\right) .
$$

Proof. As in the proof of Proposition 3.1, the $\mathrm{C}^{*}$-algebra $\left\langle X X^{*}\right\rangle$ is nondegenerately represented on $K$. Let $\left(e_{\alpha}\right)$ be a contractive approximate identity in $\left\langle X X^{*}\right\rangle$. We may suppose that each $e_{\alpha} \in \operatorname{span}\left(X X^{*}\right)$. Moreover, note that $e_{\alpha} \rightarrow 1_{K}$ in the weak* topology due to nondegeneracy. When $u \in \operatorname{span}\left(T T^{*}\right)$, the net $\left(u e_{\alpha}\right)$ satisfies the statement. The general case of $\left(T T^{*}\right)^{\prime \prime}$ follows by weak* approximation, using the Kaplansky theorem for the inclusion $\operatorname{span}\left(T T^{*}\right) \subset\left(T T^{*}\right)^{\prime \prime}$.

As for the final statements, let $\left(f_{\beta}\right) \subseteq \operatorname{span}\left(X^{*} X\right)$ be a contractive approximate identity for $\left\langle X^{*} X\right\rangle$. Now we may continue as above, using the nets $\left(f_{\beta} u\right)$ for $u \in \operatorname{span}\left(T^{*} T\right)$ and

$$
\left(\begin{array}{cc}
1_{K} & 0 \\
0 & f_{\beta}
\end{array}\right) v\left(\begin{array}{cc}
e_{\alpha} & 0 \\
0 & 1_{H}
\end{array}\right) \quad \text { for } \quad v \in\left(\begin{array}{cc}
\operatorname{span}\left(T T^{*}\right) & T \\
T^{*} & \operatorname{span}\left(T^{*} T\right)
\end{array}\right) .
$$

We are ready to formulate the main theorem of this section. Note that the result does not immediately follow from Theorem 2.1 as the $W^{*}$-sub-TRO $X$ need not satisfy the stronger nondegeneracy condition of Theorem 2.1 (i.e. nondegeneracy with respect to the norm topology).

Theorem 3.3. Assume that $X$ and $T$ are $W^{*}-T R O s, X$ is nondegenerate in $T$ (in the $W^{*}$-TRO sense) and that $P: T \rightarrow X$ is a normal (i.e. weak ${ }^{*}$-continuous) contractive surjective idempotent map. Then $P$ extends to a normal conditional expectation from $\mathrm{R}_{T}$ onto $\mathrm{R}_{X}$, where $\mathrm{R}_{T}=\mathrm{A}_{T}^{\prime \prime}$ and $\mathrm{R}_{X}=\mathrm{A}_{X}^{\prime \prime}$. 
Proof. As before, we use Theorem 2.5 of [EOR01] to observe that $P$ is a TRO conditional expectation.

Define $P P^{\dagger}$ on $\operatorname{span}\left(T X^{*}\right)$ as in the $C^{*}$-algebra case (see Theorem 2.11). Consider a net $\left(a_{\alpha}\right)$ in $\operatorname{span}\left(T X^{*}\right)$ converging weak* to $a \in \operatorname{span}\left(T X^{*}\right)$. Write $a_{\alpha}=$ $\sum_{i=0}^{n_{\alpha}} b_{i}^{\alpha}\left(x_{i}^{\alpha}\right)^{*}$ and $a=\sum_{i=0}^{n} b_{i}\left(x_{i}\right)^{*}$ where $n_{\alpha} \in \mathbb{N}, b_{i}^{\alpha} \in T, x_{i}^{\alpha} \in X$, etc.. Due to nondegeneracy, functionals $\omega_{y \xi, \eta}$ with $y \in X, \xi \in H$ and $\eta \in K$ are linearly dense in $\mathrm{B}(K)_{*}$. Since $P$ is normal,

$$
\begin{aligned}
\omega_{y \xi, \eta}\left(P P^{\dagger}\left(a_{\alpha}\right)\right) & =\sum_{i=0}^{n_{\alpha}}\left(P\left(b_{i}^{\alpha}\right)\left(x_{i}^{\alpha}\right)^{*} y \xi \mid \eta\right)=\left(P\left(\sum_{i=0}^{n_{\alpha}} b_{i}^{\alpha}\left(x_{i}^{\alpha}\right)^{*} y\right) \xi \mid \eta\right) \\
& \rightarrow\left(P\left(\sum_{i=0}^{n} b_{i}\left(x_{i}\right)^{*} y\right) \xi \mid \eta\right)=\omega_{y \xi, \eta}\left(P P^{\dagger}(a)\right) .
\end{aligned}
$$

By approximation, we see that $P P^{\dagger}$ is weak* continuous on bounded subsets of $\operatorname{span}\left(T X^{*}\right)$ (note that, as in the proof of Theorem 2.1. $P P^{\dagger}$ is contractive on $\left.\operatorname{span}\left(T X^{*}\right)\right)$.

Extend $P P^{\dagger}$ to $\left(T T^{*}\right)^{\prime \prime}$, which is the weak* closure of $\operatorname{span}\left(T X^{*}\right)$, by defining

$$
\widetilde{P P^{\dagger}}(u)=\mathrm{w}^{*}-\lim P P^{\dagger}\left(a_{\alpha}\right)
$$

where $\left(a_{\alpha}\right) \subseteq \operatorname{span}\left(T X^{*}\right)$ is bounded and converges to $u$ in the weak ${ }^{*}$ topology (such a net exists by Lemma 3.2). First note that the extension is well-defined: a weak* cluster point of $\left(P P^{\dagger}\left(a_{\alpha}\right)\right)$ always exists due to boundedness, and if $\left(b_{\beta}\right)$ is another net satisfying the requirements, then $a_{\alpha}-b_{\beta} \rightarrow 0$ weak* in $\operatorname{span}\left(T X^{*}\right)$ (joint net with the natural direction), and since $\left(a_{\alpha}-b_{\beta}\right)$ is bounded and $P P^{\dagger}$ is weak* continuous on bounded sets, it follows that $\mathrm{w}^{*}-\lim P P^{\dagger}\left(a_{\alpha}\right)=\mathrm{w}^{*}-\lim P P^{\dagger}\left(b_{\beta}\right)$.

Next we show that the extension is weak* continuous on bounded sets. Let $\left(u_{\alpha}\right)$ be a bounded net in $\left(T T^{*}\right)^{\prime \prime}$ converging weak* to $u$. For every finite set $F \subseteq \mathrm{B}(K)_{*}$, every natural number $n$ and every $\alpha$, choose $a_{\alpha}^{n, F}$ in $\operatorname{span}\left(T X^{*}\right)$ with $\left\|a_{\alpha}^{n, F}\right\| \leq\left\|u_{\alpha}\right\|$ such that for every $\omega \in F$

$$
\omega\left(a_{\alpha}^{n, F}-u_{\alpha}\right)<1 / n
$$

(note the use of Lemma 3.2 in the choice of $a_{\alpha}^{n, F}$ ). Then $\mathrm{w}^{*}-\lim _{(n, F)} a_{\alpha}^{n, F}=u_{\alpha}$ and $\mathrm{w}^{*}-\lim _{(\alpha, n, F)} a_{\alpha}^{n, F}=u$. By definition,

$$
\widetilde{P P^{\dagger}}\left(u_{\alpha}\right)=\mathrm{w}^{*}-\lim _{(n, F)} P P^{\dagger}\left(a_{\alpha}^{n, F}\right) \quad \text { and } \widetilde{P P^{\dagger}}(u)=\mathrm{w}^{*}-\lim _{(\alpha, n, F)} P P^{\dagger}\left(a_{\alpha}^{n, F}\right),
$$

and hence

$$
\begin{aligned}
\mathrm{w}^{*}-\lim _{\alpha} \widetilde{P P^{\dagger}}\left(u_{\alpha}\right) & =\mathrm{w}^{*}-\lim _{\alpha} \mathrm{w}^{*}-\lim _{(n, F)} P P^{\dagger}\left(a_{\alpha}^{n, F}\right) \\
& =\mathrm{w}^{*}-\lim _{(\alpha, n, F)} P P^{\dagger}\left(a_{\alpha}^{n, F}\right)=\widetilde{P P^{\dagger}}(u) .
\end{aligned}
$$

Thus $\widetilde{P P^{\dagger}}$ is weak* continuous on bounded sets.

We next claim that $\overline{P P^{\dagger}}$ is contractive. Let $u \in\left(T T^{*}\right)^{\prime \prime}$ and apply Lemma 3.2 to obtain a net $\left(a_{\alpha}\right)$ in $\operatorname{span}\left(T X^{*}\right)$, bounded by $\|u\|$, converging weak* to $u$. For every $\omega \in \mathrm{B}(K)_{*}$,

$$
\left|\omega\left(\widetilde{P P^{\dagger}}(u)\right)\right|=\lim _{\alpha}\left|\omega\left(P P^{\dagger}\left(a_{\alpha}\right)\right)\right| \leq \lim _{\alpha}\left\|a_{\alpha}\right\|\|\omega\| \leq\|u\|\|\omega\| .
$$

That is, $\widetilde{P P^{\dagger}}$ is contractive. 
It is easy to check that $\widetilde{P P^{\dagger}}$ is a projection onto $\left(X X^{*}\right)^{\prime \prime}$. Indeed, $\widetilde{P P^{\dagger}}$ agrees with the identity map on $\operatorname{span}\left(X X^{*}\right)$, and then using the Kaplansky density theorem and the fact that $\widetilde{P P^{\dagger}}$ is weak* continuous on bounded sets, we obtain the claim. Combined with contractivity, we see that $\widetilde{P P^{\dagger}}$ is a conditional expectation and thus in particular is positive.

By the order-theoretic characterisation of normality (see for example Con00, Definition 46.1 and Proposition 43.1]), positivity and weak* continuity on bounded sets imply that $\widetilde{P P^{\dagger}}$ is normal. So $\widetilde{P P^{\dagger}}$ is a normal conditional expectation. A similar argument produces a normal conditional expectation $\widetilde{P^{\dagger} P}$, and as in the earlier case, we form the the matrix map from $\mathrm{R}_{T}$ onto $\mathrm{R}_{X}$.

We still need to check that the matrix map is contractive. As in the proof of Theorem 2.1, we see that the map

$$
\left(\begin{array}{cc}
P P^{\dagger} & P \\
P^{\dagger} & P^{\dagger} P
\end{array}\right):\left(\begin{array}{cc}
\operatorname{span}\left(T X^{*}\right) & T \\
T^{*} & \operatorname{span}\left(X^{*} T\right)
\end{array}\right) \rightarrow\left(\begin{array}{cc}
\operatorname{span}\left(X X^{*}\right) & X \\
X^{*} & \operatorname{span}\left(X^{*} X\right)
\end{array}\right)
$$

is contractive. Using Lemma 3.2 again, we may apply the same argument as in the case of $\widetilde{P P^{\dagger}}$ to see that

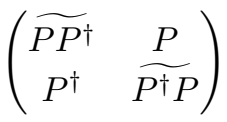

is contractive.

Acknowledgement. The work on this paper started during the visit of the secondnamed author to the University of Oulu in May 2012. We thank the Finnish Academy of Science and Letters, Vilho, Yrjö and Kalle Väisälä Foundation, for making this visit possible. The first-named author was also supported by the Emil Aaltonen Foundation. The second-named author was partly supported by the National Science Centre (NCN) grant no. 2011/01/B/ST1/05011. We thank the referee for a careful and thoughtful reading of our manuscript.

\section{REFERENCES}

[Con00] J. B. Conway, A course in operator theory, American Mathematical Society, Providence, RI, 2000.

[EOR01] E. G. Effros, N. Ozawa, and Z.-J. Ruan, On injectivity and nuclearity for operator spaces, Duke Math. J. 110 (2001), 489-521.

[Ham99] M. Hamana, Triple envelopes and Šilov boundaries of operator spaces, Math. J. Toyama Univ. 22 (1999), 77-93.

[KaRu02] M. Kaur and Z-J. Ruan, Local properties of ternary rings of operators and their linking $C^{*}$-algebras, J. Funct. Anal. 195 (2002), no. 2, 262-305.

[NeRu02] M. Neal and B. Russo, Operator space characterizations of $C^{*}$-algebras and ternary rings, Pacific J. Math. 209 (2003), no. 2, 339-364.

[NSSS12] M. Neufang, P. Salmi, A. Skalski and N. Spronk, Contractive idempotents on locally compact quantum groups, Indiana Univ. Math. J., to appear, available at arXiv:1209.6508.

[You83] M. A. Youngson, Completely contractive projections on $C^{*}$-algebras, Quart. J. Math. Oxford Ser. (2) 34 (1983), 507-511. 
Department of Mathematical Sciences, University of Oulu, PL 3000, FI-90014 Oulun YLIOPISTO, FINLAND

E-mail address: pekka.salmi@iki.fi

Institute of Mathematics of the Polish Academy of Sciences, ul.Śniadeckich 8, 00-956 WarszaWa, POland

E-mail address: a.skalski@impan.pl 